\title{
C4d-expressing glomerulopathy and proteinuria post transplantation of a too-big-for-size mismatched kidney allograft: An unusual case with good outcome
}

\author{
Francois Gougeon ${ }^{1}$, Alexei V. Mikhailov ${ }^{1}$, Keisha Gibson², Tomasz Kozlowski ${ }^{3}$, \\ Harsharan K. Singh ${ }^{1}$, and Volker Nickeleit ${ }^{1}$ \\ ${ }^{1}$ Department of Pathology and Laboratory Medicine, Division of Nephropathology, \\ ${ }^{2}$ Department of Medicine and Pediatrics, Division of Nephrology, and \\ ${ }^{3}$ Department of Surgery, Division of Abdominal Transplant, The University \\ of North Carolina, Chapel Hill, NC, USA
}

Key words biopsy - C4d - glomerulopathy - proteinuria size mismatch - transplantation
Received

July 13, 2017;

accepted

July 26, 2017

Correspondence to Volker Nickeleit, MD University of North Carolina, Division of Nephropathology, 160 N Medical Dr, Brinkhous-Bullitt Building, Room 409, Campus Box 7525, Chapel Hill, NC, 27599-7525, USA volker_nickeleit@ med.unc.edu
Abstract. A 5-year-old severely growthretarded child with tubulointerstitial, oliguric end-stage renal disease received an adult-size kidney transplant. Three years post grafting under standard triple immunosuppression (mycophenolate mofetil, tacrolimus, and prednisone) de novo nephrotic range proteinuria without the nephrotic syndrome developed. Graft function was normal (serum creatinine: $0.2-0.3 \mathrm{mg} / \mathrm{dL}$ ), there were no donor-specific HLA antibodies (DSA), and the urine sediment was inactive. Two biopsies collected 3 and 4 years post-transplantation showed severe glomerular capillary wall remodeling and associated pseudolinear C4d staining as morphologic correlates for the proteinuria. Changes resembled those seen in so-called "size-mismatch transplant glomerulopathies". There was no evidence of a glomerulonephritis, acute or chronic rejection including transplant glomerulopathy, interstitial fibrosis, peritubular capillary $\mathrm{C} 4 \mathrm{~d}$ deposits, or multilamination of peritubular capillary basement membranes. The glomerular changes were not detected in the implantation zero-hour biopsy or the recipient's native renal biopsy. At the end of follow-up 64 months post transplantation, proteinuria persisted at subnephrotic levels in the setting of stable graft function and undetectable DSAs. This unique case adds to the list of causes of nonrejection-associated post-transplant proteinuria. It demonstrates for the first time that a too-large-for-body-size mismatched graft is associated with a presumably sheer stressinduced $\mathrm{C} 4 \mathrm{~d}$ expressing glomerulopathy, severe proteinuria, and favorable outcome.

\section{Introduction}

Proteinuria post renal transplantation constitutes a clinical alarm for adverse in- tra-graft events. Depending on the timeline and the degree of proteinuria, three major underlying etiologies can be considered: [1] acute/chronic rejection, [2] recurrent or de novo glomerular diseases (e.g., membranous glomerulopathy, FSGS), [3] other glomerulopathies (e.g., calcineurin inhibitor-induced structural toxicity). Outcome depends on the underlying etiology and is often unfavorable, such as in rejection induced transplant glomerulopathy or recurrent FSGS [1, 2, 3]. Glomerular injury and proteinuria due to transplantation of a severely size-mismatched organ is uncommon. There are previous reports of adult renal allograft recipients with too-small-for-body-size pediatric donor organs presenting with severe proteinuria. The here-described opposite clinical scenario, i.e., proteinuria and favorable outcome in a too-big-for-body-size kidney transplant showing a de-novo, complement factor C4d expressing "size mismatch" glomerulopathy, has hitherto not been described. Our case furthers knowledge of potential complications associated with transplantation of sizemismatched renal allografts [4].

\section{Case report}

The patient is an 11-year-old Caucasian female with dwarfism due to heterozygous mutation of the WDR19 gene, which is linked to cranioectodermal dysplasia 4 (OMIM - Online Mendelian Inheritance in Man: 614378), a syndrome associated with small stature and nephronophthisis. At age 


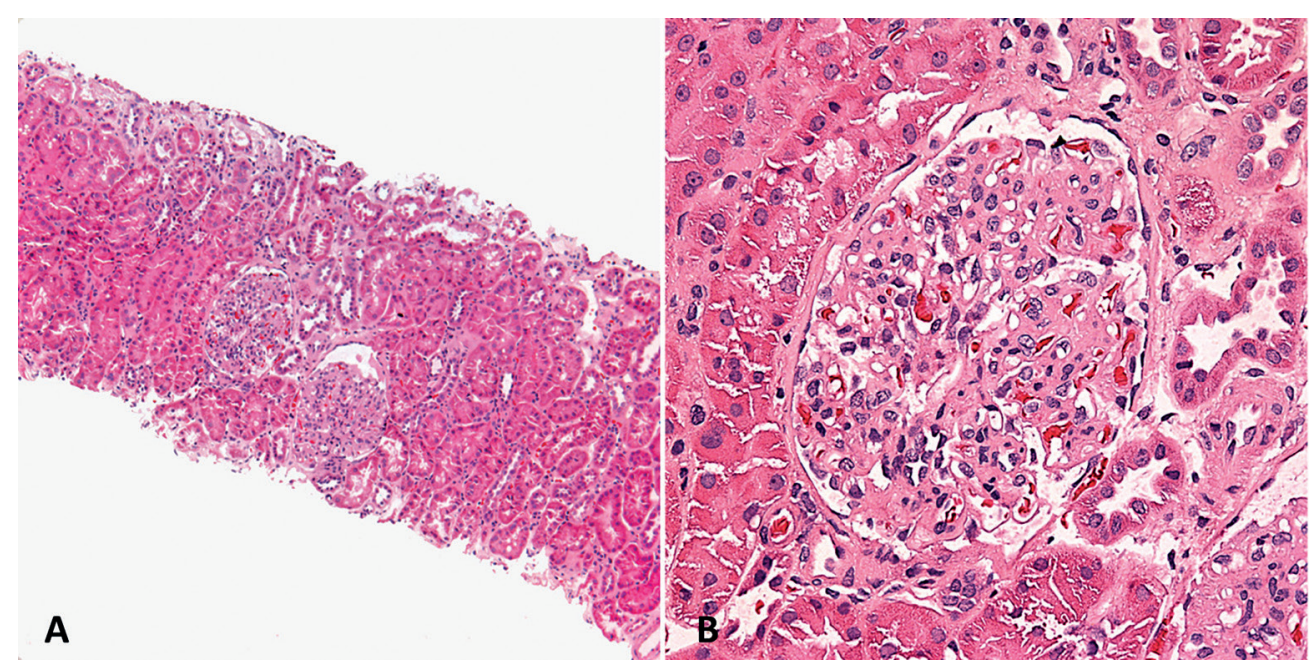

Figure 1. Second follow-up transplant biopsy (2016) showing mild mesangial matrix expansion without interstitial inflammation and only minimal focal fibrosis without tubular atrophy (H \& E stain). Identical changes were seen in the first biopsy collected in 2015. Original magnification A: $\times 200, B: \times 600$.

3 , she presented with progressive renal insufficiency. A native renal biopsy showed advanced chronic changes with $50 \%$ global glomerulosclerosis and severe interstitial fibrosis. Immunofluorescence and electron microscopic studies showed no characteristic and diagnostic glomerular abnormalities.

In 2012, at age 5, the by-then oliguric patient underwent renal transplantation with an adult-size organ from a deceased 13 -year-old male gunshot victim. The $11.8 \mathrm{~kg}$ recipient measured $88.7 \mathrm{~cm}$ in height; measurements well below the $3^{\text {rd }}$ percentile for her age. On ultrasound examination, her $4.4 \mathrm{~cm}$ and $4.7 \mathrm{~cm}$ native kidneys were echo dense with small cysts; (expected kidney length for age: $8.1 \mathrm{~cm}$ ). The donor organ measured $11.2 \mathrm{~cm}$, which is within the range of a fully matured adult kidney. To accommodate the large donor organ, the allograft was placed intraabdominally and anastomosed directly to the recipient's aorta. Native nephrectomies were not performed. A post-perfusion zero-hour allograft biopsy showed only mild acute tubular injury without glomerular abnormalities by light, electron, or immunofluorescence microscopy including unremarkable incubations for $\mathrm{C} 4 \mathrm{~d}$.

The immediate post-operative course was uneventful with good renal function (serum creatinine $(\mathrm{S}-\mathrm{Cr}$ ): $0.2-0.3 \mathrm{mg} / \mathrm{dL}$; transient evidence of hyperfiltration with $165 \mathrm{~mL} / \mathrm{min}$ estimated glomerular filtration rate (eGFR) calculated by CKiDs bed- side Schwartz Formula) under maintenance immunosuppression with mycophenolate mofetil, tacrolimus, and prednisone. During the first year of follow-up (S-Cr: $0.4 \mathrm{mg} /$ $\mathrm{dL}$; eGFR $98 \mathrm{~mL} / \mathrm{min}$ ), the patient showed minimal to mild $(1+)$ proteinuria. Proteinuria increased to moderate levels during year 2 (urine protein to creatinine ratio (UPC): 1.7; S-Cr: $0.3 \mathrm{mg} / \mathrm{dL}$; eGFR $131 \mathrm{~mL} / \mathrm{min}$ ) and reached nephrotic range 3 years post grafting (total urine protein $8.1 \mathrm{~g} / 24$ hours; nonnephritic urine sediment; S-Cr: $0.5 \mathrm{mg} / \mathrm{dL}$; eGFR: $88 \mathrm{~mL} / \mathrm{min}$ ). There was no evidence of the nephrotic syndrome, and all standard laboratory test results were within normal limits, including serum albumin and cholesterol levels. Donor-specific MHC-class I or class II antibodies were not detected (also see below). The patient was compliant with her medication with satisfactory tacrolimus trough levels. In 20153 years post transplantation, the patient underwent a diagnostic renal allograft biopsy to evaluate the cause of the proteinuria (Figure 1) (Table 1).

Light microscopy showed 16 glomeruli with mild mesangial expansion and a secondary form of segmental tuft sclerosis in 1/16 glomeruli. Capillary loops were within normal limits. The interstitium demonstrated only focal minimal fibrosis and tubular atrophy without inflammation. Blood vessels were unremarkable.

Immunofluorescence microscopy showed, along glomerular capillary walls, diffuse 
Table 1. Summary of biopsy and clinical data.

\begin{tabular}{|c|c|c|c|}
\hline & $\begin{array}{l}2012 \text { biopsy } \\
\text { (implantation) }\end{array}$ & $\begin{array}{c}2015 \text { biopsy } \\
\text { (3 years post grafting) }\end{array}$ & $\begin{array}{c}2016 \text { biopsy } \\
\text { (4 years post grafting) }\end{array}$ \\
\hline \multicolumn{4}{|l|}{ Clinical data } \\
\hline Proteinuria & $\mathrm{N} / \mathrm{A}$ & $8.1 \mathrm{~g} / 24$ hours & $4.2 \mathrm{~g} / 24$ hours \\
\hline Hematuria & $\mathrm{N} / \mathrm{A}$ & Microscopic, no casts & Microscopic, no casts \\
\hline Serum creatinine & $\mathrm{N} / \mathrm{A}$ & $0.5 \mathrm{mg} / \mathrm{dL}$ & $0.60 \mathrm{mg} / \mathrm{dL}$ \\
\hline Donor-specific HLA antibodies & $\mathrm{N} / \mathrm{A}$ & Not detected & Not detected \\
\hline Nephrotic syndrome & $\mathrm{N} / \mathrm{A}$ & Absent & Absent \\
\hline \multicolumn{4}{|l|}{ Light microscopy } \\
\hline Glomeruli & Normal & Minimal non-diagnostic changes & Minimal non-diagnostic changes \\
\hline Interstitium & $\begin{array}{c}\text { Mild acute } \\
\text { tubular injury }\end{array}$ & $\begin{array}{l}\text { Minimal interstitial fibrosis, } \\
\text { no inflammation }\end{array}$ & $\begin{array}{l}\text { Minimal interstitial fibrosis, } \\
\text { no inflammation }\end{array}$ \\
\hline Vessels & Normal & Normal, no endarteritis & Normal, no endarteritis \\
\hline \multicolumn{4}{|l|}{ Immunofluorescence } \\
\hline C4d (glomeruli) & Negative & $3+$ (global, pseudolinear) & 3-4+ (global, pseudolinear) \\
\hline C4d (PTC) & Negative & Negative & Negative \\
\hline IgM (glomeruli) & Negative & $2+$ (segmental, granular) & $2+$ (segmental, granular) \\
\hline Collagen IV; $\alpha 5$ (glomeruli) & $\mathrm{N} / \mathrm{A}$ & Normal & Normal \\
\hline \multicolumn{4}{|l|}{ Electron microscopy } \\
\hline Glomeruli & Normal & $\begin{array}{l}\text { Marked transmembranous capillary } \\
\text { wall remodeling }\end{array}$ & $\begin{array}{l}\text { Marked transmembranous capillary } \\
\text { wall remodeling }\end{array}$ \\
\hline Peritubular capillaries & Normal & Normal, no lamination & Normal, no lamination \\
\hline
\end{tabular}

global pseudolinear staining for the complement degradation product $\mathrm{C} 4 \mathrm{~d}(3+$ on a scale 0 -4), diffuse segmentally-accentuated granular staining for $\operatorname{IgM}(2+$ on a scale $0-4)$ and $\mathrm{C} 3$ (1+ on a scale $0-4)$ (Figure 2). No characteristic deposits were seen in immunofluorescence incubations to detect IgG, IgA, C1q, fibrin, $\kappa$-, and $\lambda$-light chains. Staining for collagen type $4, \alpha 5$ chain demonstrated a normal pattern. C4d staining was not detected along peritubular capillaries. The overall immunofluorescence $\mathrm{C} 4 \mathrm{~d}$ staining profile (negative along peritubular capillaries, positive along glomerular capillary walls) was confirmed by immunohistochemistry performed on formalin-fixed and paraffin-embedded tissue sections (Figure 3).

Ultrastructurally, diffuse marked irregularities of the glomerular capillary walls were found with splitting/basket weaving of the lamina densa and pronounced remodeling, accentuated under activated podocytes (Figure 4). There was no evidence of capillary wall duplication/transplant glomerulopathy, endocapillary hypercellularity, or immune complex type deposits. Peritubular capillaries did not show basement membrane multilaminations.
A diagnosis of "size-mismatch type glomerulopathy" was rendered. During followup, patient management and therapy remained largely unchanged except for the substitution of a calcium channel blocker with a $\beta$-blocker for optimized blood pressure control. Renin angiotensin aldosterone system blocking agents were not administered due to a patient history of adverse reactions.

In 2016, 4 years after transplantation, a second follow-up renal biopsy was performed to re-evaluate the cause of persistent nephrotic range proteinuria and a bump in S-Cr levels $(4.2 \mathrm{~g} / 24$ hours; S-Cr: $0.6 \mathrm{mg} / \mathrm{dL}$; eGFR $75 \mathrm{~mL} / \mathrm{min}$ ). All other clinical and laboratory parameters had remained unchanged. The follow-up biopsy showed light, immunofluorescence, and electron microscopic changes similar to those noted in the biopsy from 2015 (Figures 1, 2, 3, 4) (Table 1); there was no rejection and no increase in chronic injury. Postbiopsy therapy remained unchanged.

At last follow-up, 64 months post grafting, the 11-year-old still severely growthretarded patient presented with persistent although decreased proteinuria (UP/C of 1.3, urine albumin to creatinine ratio $(\mathrm{UA} / \mathrm{C})$ of 

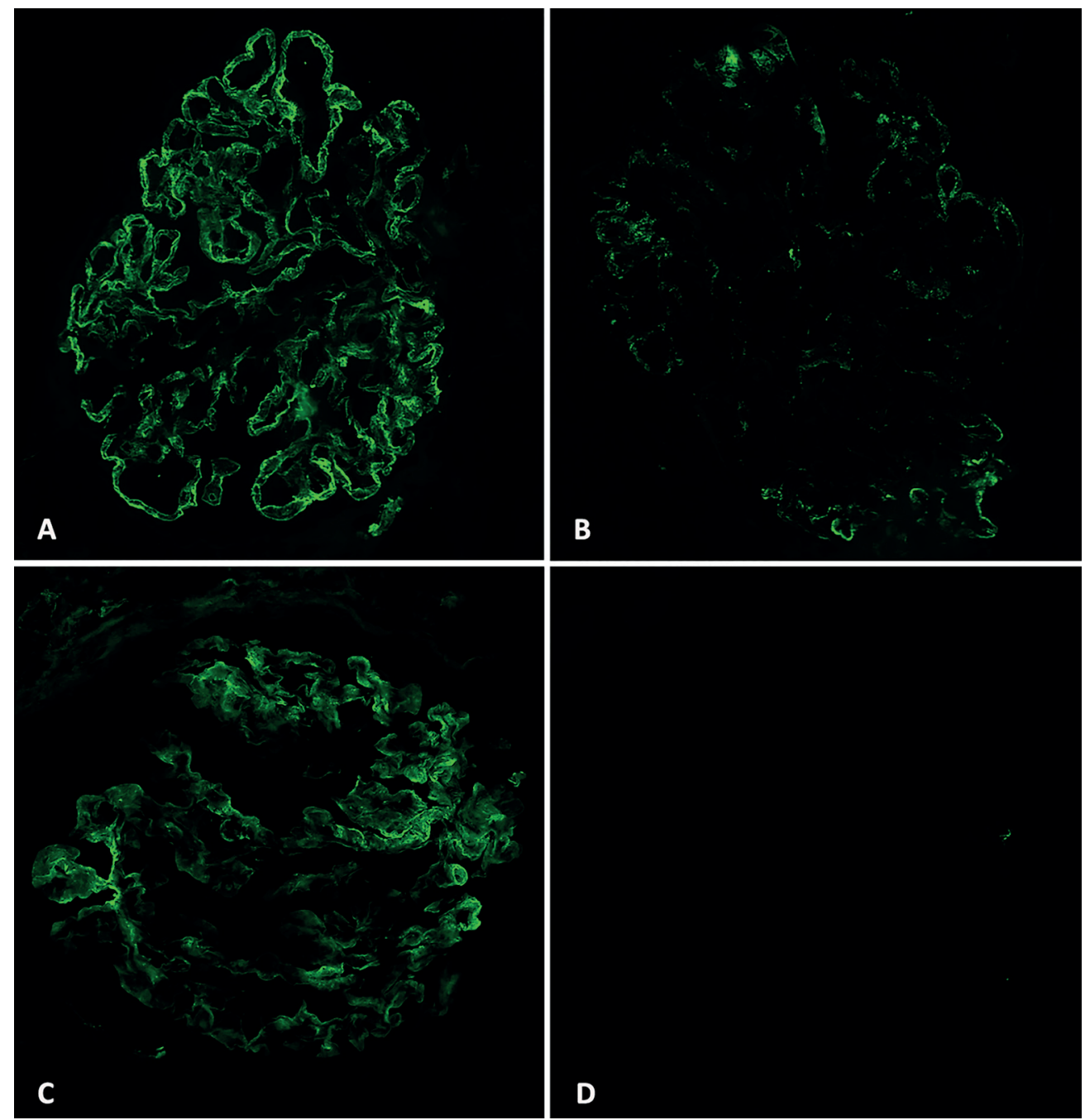

Figure 2. A, B: Second follow-up allograft biopsy from 2016 (very similar results were seen in the first biopsy from 2015). Staining for the complement degradation product C4d (A) demonstrates a strong pseudolinear global signal along glomerular capillary walls. In contrast, an IgM incubation (B) only shows a relatively weak, segmental, granular staining pattern. Of note: no significant glomerular staining is noted for IgG, IgA, C3, C1q, $\kappa$ - and $\lambda$-light chains; C4d is not seen along peritubular capillaries (data not illustrated). C, D: Control case of native kidney with hereditary nephropathy/Alport syndrome: This native control biopsy with hereditary nephropathy shows segmental glomerular C4d deposits (C) along glomerular capillary walls resembling those illustrated in (A); significant glomerular IgM staining is not found (D). Confocal immunofluorescence microscopy; original magnification $\times 400$.

1.1) and stable graft function (S-Cr: $0.45 \mathrm{mg} /$ dL; eGFR $106 \mathrm{~mL} / \mathrm{min}$ ). Her blood pressure remained well controlled $\left(<95^{\text {th }}\right.$ percentile for age and height). Over the entire posttransplantation period, no donor-specific MHC-class I or class II antibodies were detected (tested 14 times; MFI/mean fluorescence intensity continuously $<200$ ).

An attempt was made to follow-up on the performance of the contralateral donor kidney. Unfortunately, the organ was presumably placed with a recipient from outside of our organ-procuring organization, and data were not available.

\section{Discussion}

Proteinuria post renal transplantation can have different etiologies and often indicates a poor prognosis. In the current case, three differential diagnoses have to be considered: 1) donor-derived, transplanted hereditary nephropathy, 2) chronic rejection-induced transplant glomerulopathy, potentially due to antibody-mediated injury, and 3) a sizemismatch glomerulopathy.

Ad 1: Although ultrastructural glomerular changes bear similarities to "Alport syndrome", a donor-derived hereditary ne- 


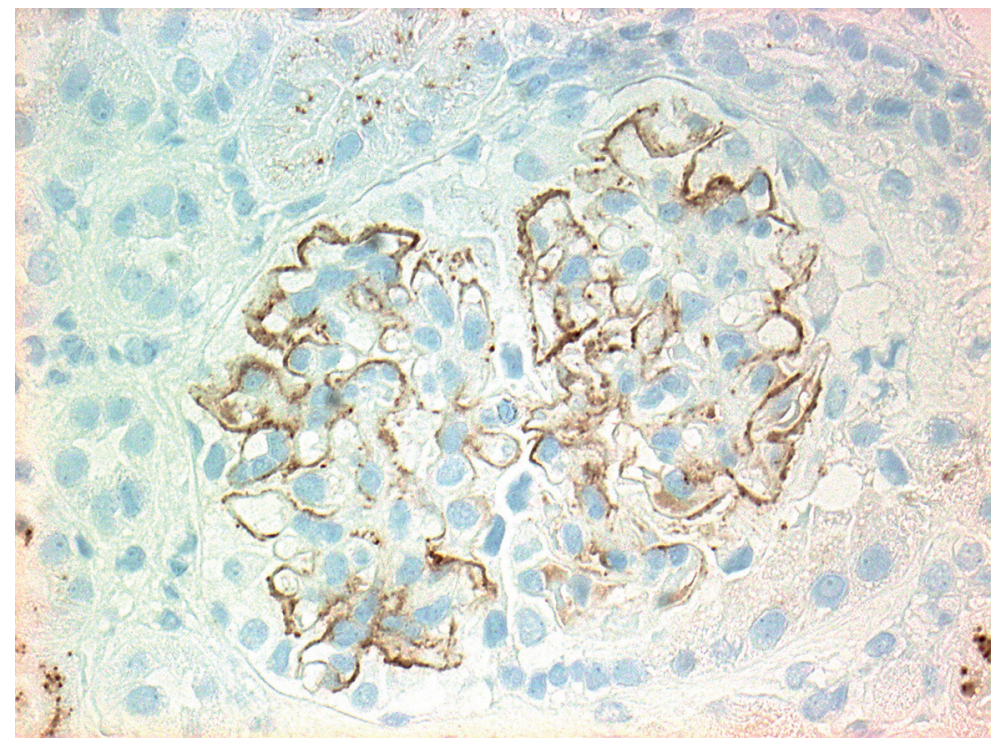

Figure 3. Second follow-up allograft biopsy from 2016 (very similar changes were seen in the first biopsy from 2015): Immunohistochemistry shows a pseudolinear staining pattern for C4d along glomerular capillary walls similar to findings illustrated in Figure 2A. Immunohistochemical incubation for C4d (Rabbit a-human polyclonal antibody, ALPCO) on formalin-fixed and paraffin-embedded tissue; original magnification $\times 400$.

phropathy can be excluded from the list of differential diagnoses based on a normal staining pattern for collagen type IV $\alpha 5$ subunit as well as normal glomeruli by light, immunofluorescence, and electron microscopy in the implantation zero-hour biopsy. This set of findings supports the notion of a de novo glomerulopathy that developed post transplantation.

Ad 2: A diagnosis of rejection-induced "transplant glomerulopathy" can also be excluded with high probability. First and foremost, the glomerulopathy displays morphologic features not known to be rejection induced, i.e., substantial subepithelial and transmembranous capillary wall remodeling rather than subendothelial GBM duplication characterizing "transplant glomerulopathy" [5]. In addition, the lack of other signs of rejection further argues against a diagnosis of "transplant glomerulopathy", i.e., no concurrent peritubular capillaritis, no $\mathrm{C} 4 \mathrm{~d}$ staining along peritubular capillaries, no tubulointerstitial or vascular rejection, no transplant glomerulitis, and no multilamination of peritubular capillary basement membranes that is typical for chronic antibody-mediated rejection (ABMR) [6]. HLA donor-specific antibodies were not detected on repeat testing. Although we cannot totally exclude the remote possibility of injury caused by circulating non-HLA donor-specific antibodies, we think that the overall presentation pattern makes this differential diagnosis unlikely.

Ad 3: Based on the clinical scenario and morphology, the diagnosis of a "size-mismatch glomerulopathy" is most likely. Organ size mismatch, i.e., typically a too-small-forbody-size transplant as a cause for allograft dysfunction, proteinuria, and glomerular remodeling was first described by Truong et al. in 1991 [7]. Truong's observations were subsequently further characterized by Nadasdy et al. and Choung et al. [8, 9], reporting glomerulopathies in $9 \%$ of adult patients receiving donor kidneys from patients younger than 10 years of age. The authors described marked alterations of the glomerular filtration barrier very similar to the currently made observations. The previously-reported
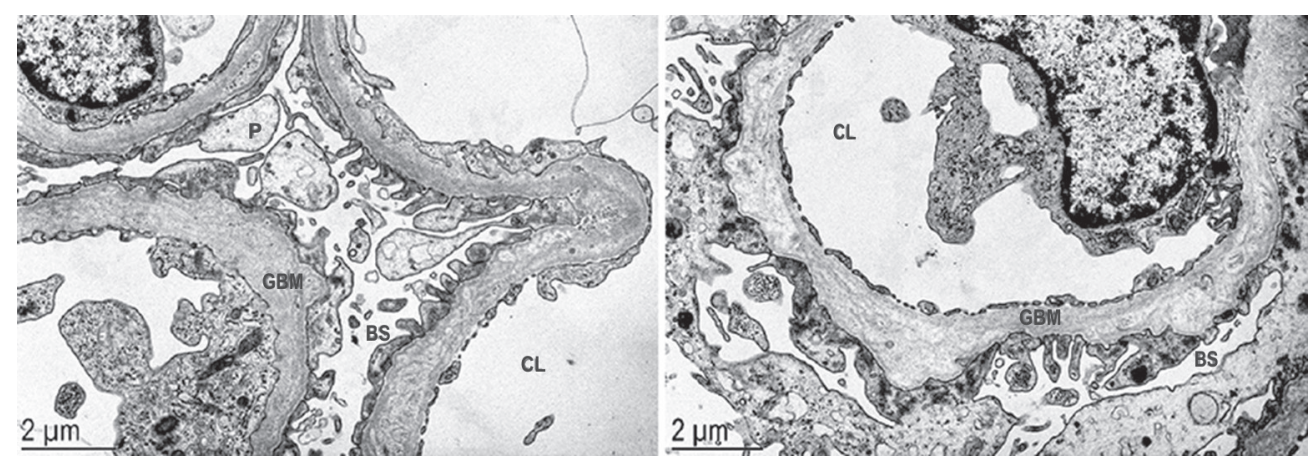

Figure 4. Electron microscopy of the 2015 and 2016 biopsies shows marked transmembranous remodeling of the glomerular capillary walls with basket-weaving of the lamina densa. Podocytes and endothelial cells are segmentally activated. There is no evidence of transplant glomerulopathy, i.e., no subendothelial duplication of glomerular basement membranes. $\mathrm{CL}$ = capillary lumen; GBM = glomerular basement membrane; $\mathrm{BS}=$ bowman's space; $\mathrm{P}=$ podocyte (transmission electron microscopy, uranyl acetate staining). Original magnification $\times 5,000, \times 10,000$. 
patients presented with significant proteinuria and, in some instances, with "elevated" serum creatinine levels or graft failure [10, $11,12,13,14,15,16,17,18]$. Glomerular changes in these small donor organs transplanted into adult recipients were postulated to be the result of "extreme graft adaptation" in the setting of "high demand" and hyperfiltration. Findings in our case fit into the spectrum of "size-mismatch glomerulopathies", however, there is one major difference: our pediatric patient received a too-big-forbody-size donor kidney.

Pathophysiologic events governing capillary wall remodeling in the current case remain hypothetical, with transient glomerular hyperfiltration post transplantation as one potential causative event. We speculate that when an adult-size kidney is grafted into a small pediatric recipient, severe alterations of blood flow and intracapillary pressure inflict "stress" on the glomerular capillary walls and podocytes resulting in remodeling and changes to the filtration barrier. In our patient, likely protracted growth retardation with lack of "catch-up growth" generated a special "intraglomerular microenvironment" promoting GBM changes that were not reported in a recent series [19].

Our patient was followed for 64 months. During this period persistent proteinuria did not lead to chronic graft damage, and renal function remained stable suggesting a favorable mid-term prognosis. Whether long-term graft survival is affected remains to be determined.

Unusual (although not entirely unexpected) was the detection of isolated pseudolinear C4d staining along glomerular capillary walls not associated with $\mathrm{C} 4 \mathrm{~d}$ deposits along peritubular capillaries or diagnostic signs of ABMR. No other significant glomerular immunoglobulin or complement factor deposits were noted. Previous reports have illustrated that such isolated pseudolinear glomerular C4d staining is associated with structural capillary wall remodeling in native and transplant kidneys independent of the underlying triggering event and independent of antibody-mediated graft injury/rejection [20, $21,22]$. We interpret our current findings along the same line and view the isolated glomerular $\mathrm{C} 4 \mathrm{~d}$ accumulation as a reflection of the pronounced capillary wall restructur- ing - and not as a sign of antibody-induced injury. This notion is further supported by two other observations: 1) At the University of Basel in Switzerland, identical findings, including isolated glomerular C4d staining, were noted in a size-mismatch glomerulopathy post transplantation of a too-small-forbody size donor kidney lacking any sign of rejection [M.J. Mihatsch, H. Hopfer, personal communication]. 2) Cases of hereditary nephropathy/Alport syndrome in native kidneys that bear morphologic similarities to size-mismatch glomerulopathies can show pseudolinear glomerular C4d staining (Figure 2; unpublished personal observation). Whether complement activation via the lectin-pathway in glomerular capillary walls undergoing remodeling causes $\mathrm{C} 4 \mathrm{~d}$ deposits needs further evaluation in future studies.

In summary, this case describes for the first time "benign" nephrotic-range proteinuria due to a de-novo C4d-expressing glomerulopathy in a too-big-for-size mismatched kidney transplant. Follow-up demonstrated good allograft function over 64 months. Glomerular injury, capillary wall remodeling, and C4d accumulation are presumably caused by adaptive hemodynamic changes/stress including the activation of the lectin pathway. Since adult kidneys represent a crucial source of donor organs in pediatric patients [19], the current case provides further insight into possible complications encountered in severely sizemismatched renal allografts.

\section{Acknowledgment}

We thank Professor Thomas Fehr, ZuerichChur, Switzerland, for his comments and critical review of the manuscript. Dr. Francois Gougeon is supported by a grant from the "Fondation du CHUM" of the University of Montreal University Hospital during his clinical fellowship at The University of North Carolina.

\section{Funding}

This research received no specific grant from any funding agency in the public, commercial, or nonprofit sector. Funding was provided by the UNC Division of Nephropathology. 


\section{Conflict of interest}

The authors certify that they have no affiliations with or involvement in any organization or entity with any financial interest or nonfinancial interest in the subject matter or materials discussed in this manuscript.

\section{References}

[1] Schachter ME, Monahan M, Radhakrishnan J, Crew J, Pollak M, Ratner L, Valeri AM, Stokes $M B$, Appel GB. Recurrent focal segmental glomerulosclerosis in the renal allograft: single center experience in the era of modern immunosuppression. Clin Nephrol. 2010; 74: 173-181. CrossRef PubMed

[2] Patri P, Seshan SV, Matignon M, Desvaux D, Lee JR, Lee J, Dadhania DM, Serur D, Grimbert P, Hartono $C$, Muthukumar $T$. Development and validation of a prognostic index for allograft outcome in kidney recipients with transplant glomerulopathy. Kidney Int. 2016; 89: 450-458. $\underline{\text { Cross- }}$ Ref PubMed

[3] Gloor JM, Sethi S, Stegall MD, Park WD, Moore $S B$, DeGoey S, Griffin MD, Larson TS, Cosio FG. Transplant glomerulopathy: subclinical incidence and association with alloantibody. Am J Transplant. 2007; 7: 2124-2132. CrossRef PubMed

[4] Miller AJ, Kiberd BA, Alwayn IP, Odutayo A, Tennankore $K K$. Donor-Recipient Weight and Sex Mismatch and the Risk of Graft Loss in Renal Transplantation. Clin J Am Soc Nephrol. 2017; 12: 669-676. CrossRef PubMed

[5] Nickeleit V, Mengel M, Colvin RB. Renal Transplant Pathology. In: Jennette JC, Olson JL, Silva FG, D'Agati VD (eds). Heptinstall's Pathology of the Kidney. 7th ed. Philadelphia, Baltimore, New York: Wolters Kluwer; 2015. p. 1321-1459.

[6] Liapis G, Singh HK, Derebail VK, Gasim AM, Kozlowski $T$, Nickeleit $V$. Diagnostic significance of peritubular capillary basement membrane multilaminations in kidney allografts: old concepts revisited. Transplantation. 2012; 94: 620-629. CrossRef PubMed

[7] Truong LD, Yoshikawa Y, Mawad J, Lederer E. Electron microscopic study of an unusual posttransplant glomerular lesion. Arch Pathol Lab Med. 1991; 115: 382-385. PubMed

[8] Nadasdy T, Abdi R, Pitha J, Slakey D, Racusen L. Diffuse glomerular basement membrane lamellation in renal allografts from pediatric donors to adult recipients. Am J Surg Pathol. 1999; 23: 437-442. CrossRef PubMed

[9] Choung HY, Meleg-Smith S. Glomerulopathy in adult recipients of pediatric kidneys. Ultrastruct Pathol. 2014; 38: 141-149. CrossRef PubMed

[10] Hayes JM, Steinmuller DR, Streem SB, Novick $A C$. The development of proteinuria and focalsegmental glomerulosclerosis in recipients of pediatric donor kidneys. Transplantation. 1991; 52: 813-817. CrossRef PubMed

[11] Yamakawa T, Kobayashi A, Yamamoto I, Nakada Y, Mafune A, Katsumata H, Furuya M, Koike K, Miki J, Yamada H, Tanno Y, Ohkido I, Tsuboi N, Yokoyama K, Yamamoto H, Yokoo T. Clinical and pathological features of donor/recipient body weight mismatch after kidney transplantation.
Nephrology (Carlton). 2015; 20 (Suppl 2): 36-39. CrossRef PubMed

[12] Amante AJ, Piñon-Barretto SC. The correlation of renal allograft weight to metabolic index ratios and glomerular filtration rate among living-unrelated kidney transplant patients: a cross-sectional study. Transplant Proc. 2008; 40: 2313-2318. CrossRef PubMed

[13] Poggio ED, Hila S, Stephany B, Fatica R, Krishnamurthi $V$, del Bosque C, Goldfarb D, Herts B, Dennis VW, Heeger PS, Braun W. Donor kidney volume and outcomes following live donor kidney transplantation. Am J Transplant. 2006; 6: 616-624. CrossRef PubMed

[14] McGee J, Magnus JH, Islam TM, Jaffe BM, Zhang R, Florman SS, et al. Donor-recipient gender and size mismatch affects graft success after kidney transplantation. J Am Coll Surg. 2010; 210: 725-726. PubMed

[15] Dick AA, Mercer LD, Smith JM, McDonald RA, Young B, Healey PJ. Donor and recipient size mismatch in adolescents undergoing living-donor renal transplantation affect long-term graft survival. Transplantation. 2013; 96: 555-559. CrossRef PubMed

[16] Goldberg RJ, Smits G, Wiseman AC. Long-term impact of donor-recipient size mismatching in deceased donor kidney transplantation and in expanded criteria donor recipients. Transplantation. 2010; 90: 867-874. CrossRef PubMed

[17] Balachandran VP, Aull MJ, Goris M, Figueiro J, Leeser DB, Kapur S. Successful transplantation of single kidneys from pediatric donors weighing less than or equal to $10 \mathrm{~kg}$ into standard weight adult recipients. Transplantation. 2010; 90: 518522. CrossRef PubMed

[18] Al-Bader WE-R, Landsberg D, Manson AD, Levin $A$. Renal function changes over time in adult recipients of small pediatric kidneys. Evidence against hyperperfusion injury. Transplantation. 1996; 62: 611-615. CrossRef PubMed

[19] Luijten JCHBM, Voet M, de Gier RPE, Nusmeier A, Scharbatke H, van der Vliet JA, Cornelissen $E A M$. Transplantation of adult living donor kidneys in small children, a single-centre initial experience. Transpl Int. 2017; 30: 640-642. CrossRef PubMed

[20] ChuaJS, Baelde HJ, Zandbergen M, Wilhelmus $S$, van Es LA, de Fijter JW, Bruijn JA, Bajema IM, Cohen D. Complement Factor C4d Is a Common Denominator in Thrombotic Microangiopathy. J Am Soc Nephrol. 2015; 26: 2239-2247. CrossRef PubMed

[21] Gasim AH, Chua JS, Wolterbeek R, Schmitz J, Weimer E, Singh HK, Nickeleit V. Glomerular $\mathrm{C} 4 \mathrm{~d}$ deposits can mark structural capillary wall remodelling in thrombotic microangiopathy and transplant glomerulopathy: C4d beyond active antibody-mediated injury: a retrospective study. Transpl Int. 2017; 30: 519-532. CrossRef PubMed

[22] von der Thusen JH, Maa de Louw R, Cg Bourgondien M, C Clahsen-van Groningen M. Ultrastructural changes of the glomerular basement membrane... unmasked by $\mathrm{C} 4 \mathrm{~d}$ staining. Transpl Int. 2017; 30: 945-946. CrossRef PubMed 